\title{
MicroRNA-205 regulates ubiquitin specific peptidase 7 protein expression in hepatocellular carcinoma cells
}

\author{
LIANG ZHU, RONG LIU, WEI ZHANG, SHENG QIAN and JIAN-HUA WANG \\ Department of Interventional Radiology, Zhongshan Hospital, Fudan University, Shanghai 200032, P.R. China
}

Received March 18, 2014; Accepted January 9, 2015

DOI: $10.3892 / \mathrm{mmr} .2015 .3998$

\begin{abstract}
Ubiquitin specific peptidase 7 (UPS7) has a critical role in the development and progression of cancer, at least in part, through its regulation of p53 protein stability. However, its molecular determinants remain to be elucidated. In the present study, it was identified that microRNA-205 (miR-205) may negatively regulate UPS7 protein levels through targeting its 3'-untranslated region in hepatocellular carcinoma (HCC) cells. As a result, miR-205 mimics inhibited USP7 protein levels while antisense miR-205 enhanced USP7 protein levels, thereby modulating the p53 signaling pathway and cell proliferation levels. In conclusion, the data presents a novel molecule for the dysregulated expression of USP7 in HCC, which may assist in elucidating mechanisms underlying the tumorigenesis of HCC.
\end{abstract}

\section{Introduction}

Hepatocellular carcinoma (HCC) has become the third most fatal type of neoplasm worldwide (1). An improved understanding of its molecular mechanisms may assist in identifying novel targets for therapeutics.

Ubiquitin specific peptidase 7 (UPS7), also known as the herpes simplex virus associated ubiquitin-specific protease, was initially isolated as a binding partner of the herpes simplex virus protein Vmw110/infected cell polypeptide 0 (2). Subsequent studies demonstrated that USP7 has critical roles in tumor development and progression (3). Downregulation of its expression usually contributes to oncogenic transformation and is crucial for tumor cell proliferation $(4,5)$. At the molecular level, USP7 has been identified as a key regulator of the p53 signaling pathway, through stabilizing p53 protein and preventing its degradation (6). In this regard, USP7 may act as a tumor suppressor and a deficiency in USP7 may

Correspondence to: Dr Jian-Hua Wang, Department of Interventional Radiology, Zhongshan Hospital, Fudan University, 180 Fenglin Road, Shanghai 200032, P.R. China

E-mail: wangjianhuazs@126.com

Key words: ubiquitin specific peptidase 7, hepatocellular carcinoma, microRNA-205, proliferation result in cell proliferation and an increase in genomic instability leading to mutagenesis (2,7). However, the molecular determinants of USP7 expression in human cancer remain to be elucidated.

MicroRNAs (miRNAs) are a class of small non-coding RNA molecules, which repress gene expression through translational repression or degradation $(8,9)$. In human cancer, increasing lines of evidence have indicated that miRNAs have important roles in tumor cell proliferation, metastasis and angiogenesis, through regulation of multiple signaling pathways, including the $\mathrm{p} 53$, Wnt/ $\beta$-Catenin and nuclear factor $(\mathrm{NF})-\kappa \mathrm{B}$ signaling pathways (10-12). Therefore, the aim of the present study was to investigate whether USP7 expression could be controlled by miRNAs in HCC.

\section{Materials and methods}

Human tissue samples. HCC tissues and adjacent non-tumor normal tissues were collected from patients undergoing routine therapeutic surgery at the Department of Interventional Radiology, Zhongshan Hospital (Shanghai, China), between May 2009 and July 2011. All samples were obtained with informed consent and approved by the Zhongshan Hospital Institutional Review Board.

Cell culture. HepG2 cells derived from HCC were obtained from the Cell Bank of Type Culture Collection of the Chinese Academy of Sciences (Shanghai, China). Cells were grown in Dulbecco's modified Eagle's medium (DMEM, Gibco-BRL, Shanghai, China) supplemented with $10 \%$ fetal bovine serum (Gibco-BRL) and maintained at $37^{\circ} \mathrm{C}$ in a humidified atmosphere with $5 \% \mathrm{CO}_{2}$.

Reverse transcription-quantitative polymerase chain reaction $(R T-q P C R)$. Total RNA from tissues and cells was extracted using the miRNA isolation kit (Ambion, Austin, TX, USA) according to the manufacturer's instructions. cDNA synthesis was performed for each RNA sample using a Reverse Transcription system (Promega Corporation, Madison, WI, USA), and oligo dT from the system was used to prime cDNA synthesis. Expression levels of mature miRNAs were assayed using a Taqman MicroRNA assay (Applied Biosystems, Foster City, CA, USA). RT-qPCR was performed using an Applied Biosystems 7300 Real-time PCR system and a TaqMan Universal PCR master mix (Applied Biosystems). PCR 
A

$\begin{array}{lcc}\text { miRNA } & \begin{array}{c}\text { Target } \\ \text { USP7 }\end{array} & \text { Position of USP7 3' UTR }\left(5^{\prime}-3^{\prime}\right) \\ \text { miR-329 } & & 11-17,32-38,991-997 \\ \text { miR-205 } & 458-464 \\ \text { miR-148 } & 623-630 \\ \text { miR-152 } & 624-631 \\ \text { miR-362 } & 1125-1131,1594-1600\end{array}$

B

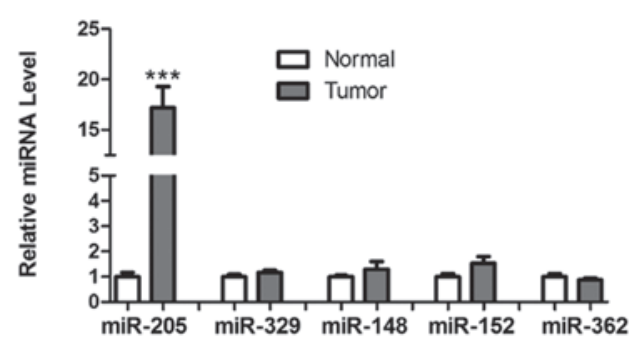

Figure 1. miR-205 is upregulated in human HCC tissues. (A) Computer prediction of miRNA binding sites in the 3'UTRs of the human USP7 gene. (B) Expression of miRNAs was determined by reverse transcription-quantitative polymerase chain reaction in human HCC tissues (tumor, $\mathrm{n}=32$ ) and adjacent noncancerous tissues (normal, $\mathrm{n}=28$ ). ${ }^{* * * *} \mathrm{P}<0.001$, compared with normal. HCC, hepatocellular carcinoma; miRNAs, microRNAs; UTR, untranslated region; USP7, ubiquitin specific peptidase 7.

A

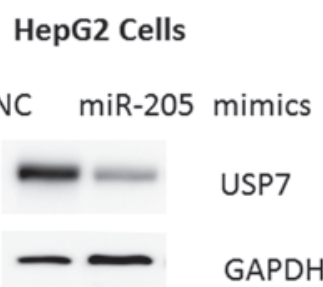

B

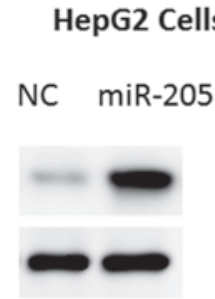

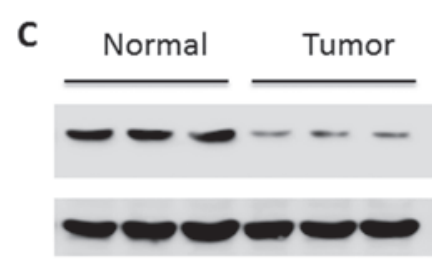

USP7

USP7

GAPDH

\section{GAPDH}

Figure 2. miR-205 regulates USP7 expression in HepG2 cells. Western blot analysis of USP7 expression in HepG2 cells transfected with (A) miR-205 mimics and (B) antisense. (C) Representative USP protein levels were determined by western blot analysis in HCC tissues (tumor) and adjacent noncancerous tissues (normal). HCC, hepatocellular carcinoma; miR, microRNA; USP7, ubiquitin specific peptidase 7.

A

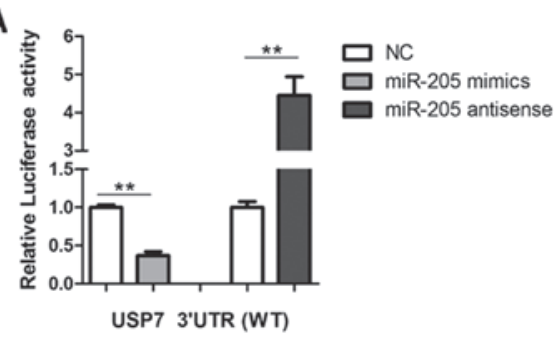

B

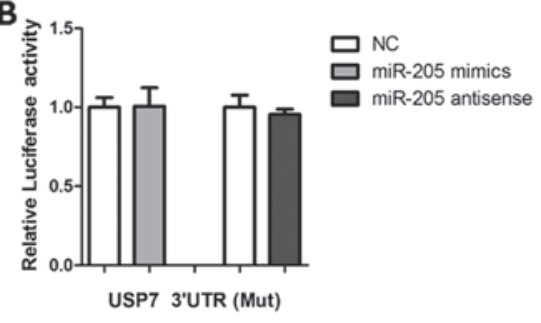

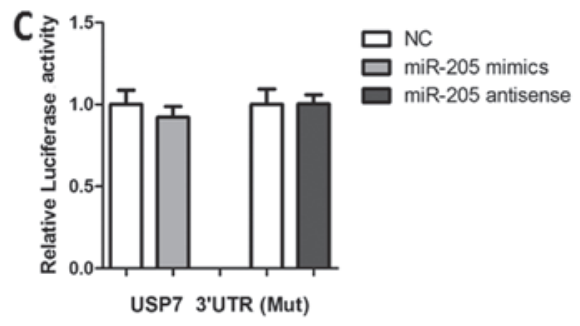

Figure 3. miR-205 targets the 3'UTR region of human USP7 gene. Luciferase reporter assays in HepG2 cells. Cells were transfected with $400 \mathrm{ng}$ of (A) WT 3'-UTR-reporter or (B) mut constructs together with miR-205 mimics, antisense or NC. ${ }^{* *} \mathrm{P}<0.01$. (C) Luciferase assays in HepG2 cells transfected with $400 \mathrm{ng}$ of the GFP-hUSP7 plasmid that lacks the USP7 3'UTR and then transfected with the indicated miRNAs. HCC, hepatocellular carcinoma; miRNAs, microRNAs; UTR, untranslated region; USP7, ubiquitin specific peptidase 7; GFP, green fluorescent protein; WT, wild-type; Mut, mutant.

conditions included an initial hold period at $95^{\circ} \mathrm{C}$ for $5 \mathrm{~min}$, followed by a two-step PCR program consisting of $95^{\circ} \mathrm{C}$ for $5 \mathrm{sec}$ and $60^{\circ} \mathrm{C}$ fo $30 \mathrm{sec}$ for 40 cycles. The primer sequences were as follows: p21 forward, 5'-TGTCCGTCAGAACCCATGC-3', reverse, 5'-CCAGCCCATGATGGTTCTGAT-3'; Bax forward, 5'-CCCGAGAGGTCTTTTTCCGAG-3', reverse, 5'-CCAGCCCATGATGGTTCTGAT-3'; and GADD45 forward, 5'-GAGAGCAGAAGACCGAAAGGA-3' and reverse, 5'-CACAACACCACGTTATCGGG-3'. All the primers were obtained from Bioyare Company (Shanghai, China). Data were collected and quantitatively analyzed accoring to the $2^{-\Delta \Delta \mathrm{Ct}}$ method. Expression of the miRNAs was normalized to that of the U6 spliceosomal RNA.

Western blot analysis. Cells were harvested and lysed with ice-cold lysis buffer (50 mM Tris- $\mathrm{HCl}, \mathrm{pH} 6.8 ; 100 \mathrm{mM}$ 2-mercaptoethanol, $2 \% \mathrm{w} / \mathrm{v} \mathrm{SDS}$ and $10 \%$ glycerol). Following centrifugation at $10,000 \mathrm{x}$ g for $15 \mathrm{~min}$ at $4{ }^{\circ} \mathrm{C}$, proteins in the supernatants were quantified and separated using 10\% SDS-PAGE. Western blot analysis was performed using the following rabbit antibodies: Anti-USP7 (1:1,000; cat. no. 3277) and p53 (1:1,000; cat. no. 9282) (polyclonal), and p21 (1:2,000; cat. no. 2947), Bax (1:1,000; cat. no. 5023) p27 (1:2,000; cat. no. 3688) and growth arrest and DNA damage 45 (GADD45; 1:1,000; cat. no. 4632) (monoclonal) (Cell Signaling Technology, Inc., Danvers, MA, USA). The secondary antibodies (1:5000, goat anti-rabbit IgG-HRP; sc-2004) were purchased from Santa Cruz Biotechnology, Inc. (Santa Cruz, CA, USA). Protein levels were normalized to total GAPDH, using a rabbit anti-GAPDH antibody (1:5,000; cat. no. H-83) (Santa Cruz Biotechnology, Inc.). The blots were visualized using chemiluminescence reagents (Amersham Pharmacia, Little Chalfont, UK).

Transfection and luciferase reporter assay. miR-205 mimics and antisense oligonucleotides were purchased from Ambion 
A

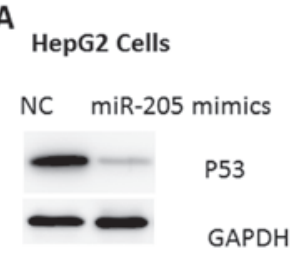

B

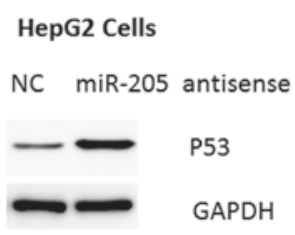

C

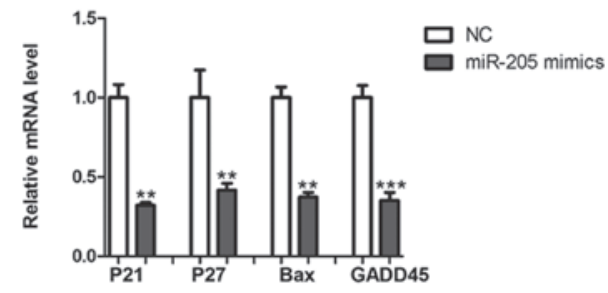

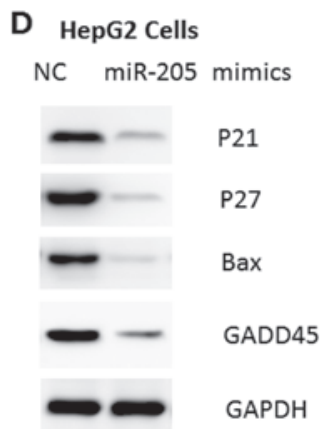

E

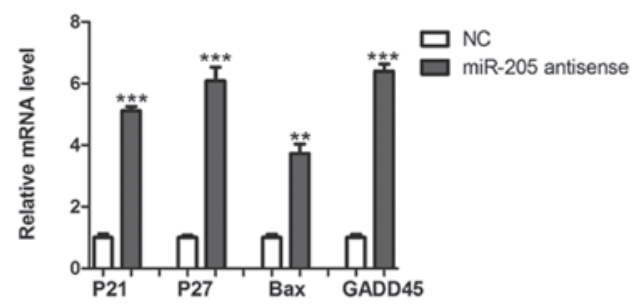

$\mathbf{F}$ HepG2 Cells NC miR-205 antisense

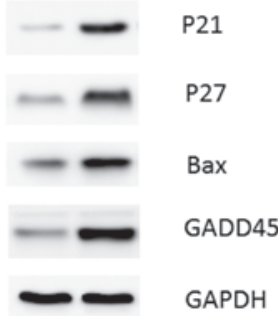

Figure 4. miR-205 regulates the p53 signaling pathway in HepG2 cells. Western blot analysis of p53 expression in HepG2 cells transfected with (A) miR-205 mimics or (B) antisense. (C) mRNA and (D) protein levels of p21, p27, Bax and GADD45 expression in HepG2 cells transfected with miR-205 mimics or NC. (E) mRNA and (F) protein levels of p21, p27, Bax and GADD45 expression in HepG2 cells transfected with miR-205 antisense or NC. NC, negative control; miRNAs, microRNAs; GADD45, growth arrest and DNA damage 45.
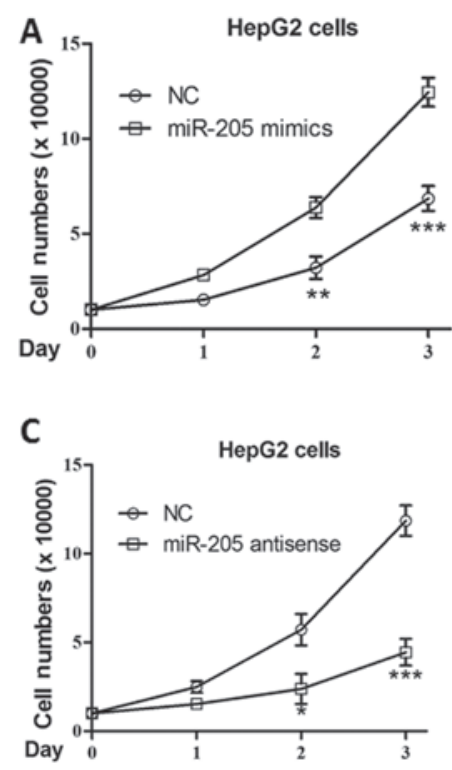

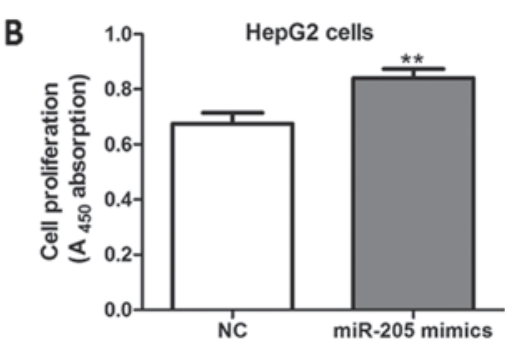

D

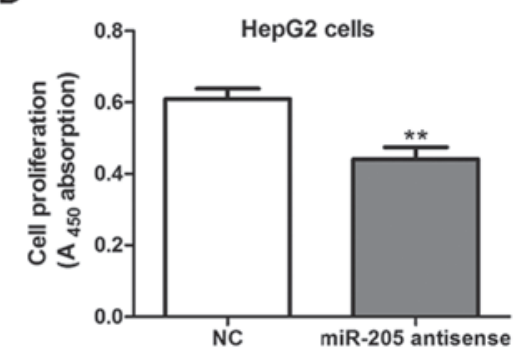

Figure 5. miR-205 controls HCC cell proliferation. (A) Growth curve and (B) cell proliferation was determined in HepG2 cells following miR-205 mimic transfection compared with NC. (C) Growth curve and (D) cell proliferation was determined in HepG2 cells following miR-205 antisense transfection compared with NC. ${ }^{*} \mathrm{P}<0.05,{ }^{* * *} \mathrm{P}<0.01$ and ${ }^{* * * *} \mathrm{P}<0.001$ compared with NC. NC, negative control; miRNAs, microRNAs; HCC, hepatocellular carcinoma.

(Invitrogen Life Technologies, Carlsbad, CA, USA). The human USP7 3'UTR was cloned into the pMir-Report miRNA expression reporter vector system (Ambion), yielding pMir-Report-USP7. Mutations were introduced in potential miR-205 binding sites using the QuikChange site-directed mutagenesis kit (Stratagene, Santa Clara, CA, USA). Transfection was performed with Lipofectamine 2000 (Invitrogen Life Technologies) according to the manufacturer's instructions. The Renilla luciferase control reporter vector pRL-TK (Promega Corporation) carrying the Renilla lucif- erase gene was used to normalize the transfection efficiency. Luciferase values were measured using the Dual-Luciferase reporter assay system (Promega Corporation).

Bromodeoxyuridine (BrdU) assays. A cell proliferation enzyme-linked immunosorbent assay (BrdU kit; Beyotime Institute of Biotechnology, Haimen, China) was used to analyze the incorporation of BrdU during DNA synthesis following the manufacturer's instructions. Absorbance was measured at $450 \mathrm{~nm}$ in the Spectra Max 190 ELISA reader 
(Molecular Devices, Sunnyvale, CA, USA). All experiments were performed in triplicate.

Statistical analysis. The data shown are presented as the mean \pm standard error of the mean of three independent experiments. Statistical analyses were conducted using GraphPad version 5.0 (GraphPad Software, Inc., La Jolla, CA, USA). Significance was analyzed using Student's t-test. $\mathrm{P}<0.05$, $\mathrm{P}<0.01$ and $\mathrm{P}<0.001$ were considered to indicate a statistically significant difference.

\section{Results}

miR-205 reduces USP7 protein expression in HepG2 cells. Using the TargetScan algorithm based on seed recognition, several miRNAs were identified, which may potentially interact with the USP7 transcript (Fig. 1A). However, only miR-205 was significantly upregulated in HCC tissues, compared with normal tissues (Fig. 1B). To assess whether miR-205 regulates USP7, miR-205 mimic or antisense were introduced into HepG2 human hepatoma cells. As a result, expression of miR-205 and anti-miR-205 reduced and increased the quantity of USP7 protein, respectively (Fig. 2A and B). Concurrent with the upregulation of miR-205, a marked reduction of USP7 protein levels was observed in HCC tissues (Fig. 2C), further suggesting that USP7 may be a target of miR-205 in HCC development.

miR-205 interacts with the 3 'UTR of USP7. To understand how miR-205 regulates USP7 expression, the luciferase reporter plasmids containing the 3'UTR of USP7 were co-transfected with miR-205 mimics or antisense oligonucleotides. As shown in Fig. 3A, miR-205 mimics led to a reduction and antisense led to an increase in luciferase activity. Furthermore, mutagenesis of the seed sequence eliminated the effects of miR-205 mimics or antisense oligonucleotides on USP7 activity (Fig. 3B). When co-expressed with a plasmid encoding green fluorescent protein-tagged human USP7 lacking the 3'UTR, neither miR-205 nor anti-miR-205 had an effect on USP7 activity (Fig. 3C). These results suggest that miR-205 binds to the 3'UTR of USP7 and reduces its protein contents.

miR-205 regulates the p53 signaling pathway in HepG2 cells. Due to the protective role of USP7 in p53 protein stability (6), it was determined whether miR-205 may affect the p53 stability. As shown in Fig. 4A, overexpression of miR-205 led to reduced p53 protein expression in HepG2 cells. Consistently, inhibition of miR-205 led to an increased expression of p53 (Fig. 4B). In addition, the downstream targets of p53 signaling, including p21, p27 and GADD45, were also regulated by miR-205 overexpression or inhibition (Fig. 4C-F).

miR-205 positively promotes cell proliferation. As the critical roles of the USP7-p53 regulatory axis is involved in cell proliferation, the effects of miR-205 on HepG2 cell growth were assessed. As a result, miR-205 mimics significantly increased cell numbers and promoted proliferation in cells post-transfection (Fig. 5A and B). Consistently, its antisense inhibited the growth of HepG2, compared with negative control-transfected cells (Fig. 5C and D).

\section{Discussion}

In the present study, it was demonstrated that miR-205 is upregulated in HCC tissues. In addition, miR-205 is able to inhibit cell proliferation in HepG2 cells, through regulation of USP7 protein levels. Therefore, for the first time, to the best of our knowledge, the present study identified that miR-205 may be an onco-microRNA involved in the progression of HCC. However, further studies are required to investigate its role in vivo.

It has been demonstrated that several miRNAs were dysregulated in HCC tissues or cell lines $(13,14)$. For instance, an in-depth analysis of miRNomes in human HCC and normal liver has been performed, which identified that miR-199a/b-3p, the third most highly expressed miRNA in the liver, was consistently decreased in HCC (15). In addition, miR-199a/b-3p may target tumor-promoting p21 protein (Cdc42/Rac)-activated kinase 4 (PAK4) to suppress HCC growth through inhibiting the PAK4/Raf/mitogen-activated protein kinase kinase/extracellular signal-regulated kinase pathway in vitro and in vivo (15). Therefore, identification of more dysregulated miRNAs may aid in improving understanding of the important deregulated miRNAs in HCC.

The roles of miR-205 have been elucidated in other types of human cancer. miR-205 promotes tumor proliferation and invasion through targeting estrogen-related receptor- $\gamma$ in endometrial carcinoma (16). In addition, miR-205 targets phosphatase and tensin homolog and PH domain and leucine rich repeat protein phosphatase 2 to augment protein kinase B signaling and drive malignant phenotypes in non-small cell lung cancer (17). However, miR-205 is frequently downregulated in prostate cancer and acts as a tumor suppressor by inhibiting tumor growth (18). Although the reason for the inconsistency remains to be elucidated at present, the effects of miR-205 may be cell- or tissue-specific.

Previous studies have demonstrated that USP7 is regulated by several signals, such as interleukin-6-mediated signal transducer and activator of transcription 3 activation $(19,20)$. However, whether its expression is controlled by miRNAs remains to be examined. Therefore, the present data on the functional interaction of miR-205 and USP7/p53 signaling in HCC may assist in elucidating mechanisms underlying tumorigenesis in HCC.

\section{References}

1. Villanueva A, Hernandez-Gea V and Llovet JM: Medical therapies for hepatocellular carcinoma: a critical view of the evidence. Nat Rev Gastroenterol Hepatol 10: 34-42, 2013.

2. Qing P, Han L, Bin L, Yan L and Ping WX: USP7 regulates the stability and function of HLTF through deubiquitination. J Cell Biochem 112: 3856-3862, 2011.

3. Nicholson B and Suresh Kumar KG: The multifaceted roles of USP7: new therapeutic opportunities. Cell Biochem Biophys 60: 61-68, 2011

4. Lee JT and $\mathrm{Gu} \mathrm{W}$ : The multiple levels of regulation by p53 ubiquitination. Cell Death Differ 17: 86-92, 2010.

5. Colland F: The therapeutic potential of deubiquitinating enzyme inhibitors. Biochem Soc Trans 38: 137-143, 2010.

6. Lee MH and Lozano G: Regulation of the p53-MDM2 pathway by 14-3-3 sigma and other proteins. Semin Cancer Biol 16: 225-234, 2006.

7. Meulmeester E, Maurice MM, Boutell C, et al: Loss of HAUSP-mediated deubiquitination contributes to DNA damage-induced destabilization of $\mathrm{Hdmx}$ and $\mathrm{Hdm} 2$. Mol Cell 18: 565-576, 2005. 
8. Sun K and Lai EC: Adult-specific functions of animal microRNAs. Nat Rev Genet 14: 535-548, 2013.

9. Ameres SL and Zamore PD: Diversifying microRNA sequence and function. Nat Rev Mol Cell Biol 14: 475-488, 2013

10. Liu Y, Xing R, Zhang X, et al: miR-375 targets the p53 gene to regulate cellular response to ionizing radiation and etoposide in gastric cancer cells. DNA Repair 12: 741-750, 2013.

11. Nagano H, Tomimaru Y, Eguchi H, et al: MicroRNA-29a induces resistance to gemcitabine through the Wnt/beta-catenin signaling pathway in pancreatic cancer cells. Int J Oncol 43: 1066-1072, 2013

12. Zhang S, Shan C, Kong G, Du Y, Ye L and Zhang X: MicroRNA-520e suppresses growth of hepatoma cells by targeting the NF-kappaB-inducing kinase (NIK). Oncogene 31: 3607-3620, 2012.

13. Giordano S and Columbano A: MicroRNAs: new tools for diagnosis, prognosis and therapy in hepatocellular carcinoma? Hepatology 57: 840-847, 2013

14. Wong CM, Kai AK, Tsang FH and Ng IO: Regulation of hepatocarcinogenesis by microRNAs. Front Biosci (Elite edi) 5: 49-60, 2013.
15. Hou J,Lin L, Zhou W, et al: Identification of miRNomes in human liver and hepatocellular carcinoma reveals miR-199a/b-3p as therapeutic target for hepatocellular carcinoma. Cancer Cell 19: 232-243, 2011.

16. Su N, Qiu H, Chen Y, Yang T, Yan Q and Wan X: miR-205 promotes tumor proliferation and invasion through targeting ESRRG in endometrial carcinoma. Oncology Reports 29: 2297-2302, 2013.

17. Cai J, Fang L, Huang Y, et al: miR-205 targets PTEN and PHLPP2 to augment AKT signaling and drive malignant phenotypes in non-small cell lung cancer. Cancer Res 73: 5402-5415, 2013.

18. Wang N, Li Q, Feng NH, et al: miR-205 is frequently downregulated in prostate cancer and acts as a tumor suppressor by inhibiting tumor growth. Asian J Androl 15: 735-741, 2013.

19. Yang Z, Huo S, Shan Y, et al: STAT3 repressed USP7 expression is crucial for colon cancer development. FEBS Lett 586: 3013-3017, 2012

20. van Loosdregt J, Fleskens V, Fu J, et al: Stabilization of the transcription factor Foxp3 by the deubiquitinase USP7 increases Treg-cell-suppressive capacity. Immunity 39: 259-271, 2013. 